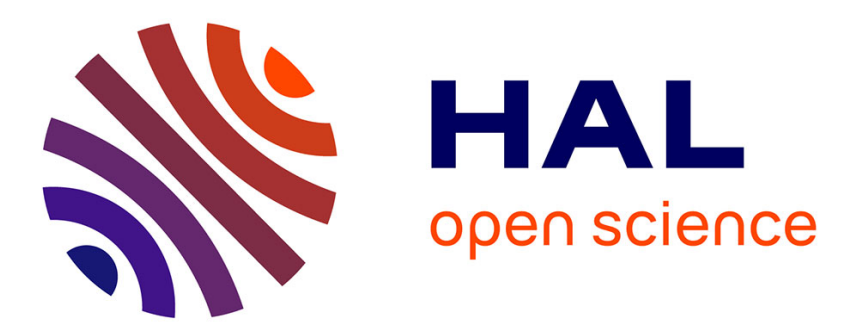

\title{
L'influence de l'activation des rapports intersexes sur les choix en faveur de la famille, du travail, du bénévolat ou des loisirs personnels
}

Manuel Tostain, Joëlle Lebreuilly

\section{- To cite this version:}

Manuel Tostain, Joëlle Lebreuilly. L'influence de l'activation des rapports intersexes sur les choix en faveur de la famille, du travail, du bénévolat ou des loisirs personnels. Pratiques Psychologiques, 2006, 12 (3), pp.331-345. 10.1016/j.prps.2006.01.013 . hal-01659272

HAL Id: hal-01659272

https://hal-normandie-univ.archives-ouvertes.fr/hal-01659272

Submitted on 8 Dec 2017

HAL is a multi-disciplinary open access archive for the deposit and dissemination of scientific research documents, whether they are published or not. The documents may come from teaching and research institutions in France or abroad, or from public or private research centers.
L'archive ouverte pluridisciplinaire $\mathbf{H A L}$, est destinée au dépôt et à la diffusion de documents scientifiques de niveau recherche, publiés ou non, émanant des établissements d'enseignement et de recherche français ou étrangers, des laboratoires publics ou privés. 


\title{
L'influence de l'activation des rapports intersexes sur les choix en faveur de la famille, du travail, du bénévolat ou des loisirs personnels
}

\section{The influence of intersexes relations activation on the choices in favour of family, work, voluntary help or personal leisures}

\author{
M. Tostain *, J. Lebreuilly \\ UFR de psychologie-PPF psychologie, bureau SE 611, \\ université de Caen-Campus 1, esplanade de la Paix, 14032 Caen cedex, France
}

\begin{abstract}
Résumé
Cette étude avait pour but d'étudier l'influence de l'activation des rapports intersexes sur les choix d'activités associées aux individualismes dit « relationnel » et " de marché ». En se fondant sur le principe d'une recherche de Marro (1998), on présentait aux sujets (des étudiants - es) des histoires dans lesquelles les protagonistes (femmes ou hommes), pour des raisons diverses (personnelles, professionnelles, etc.) doivent décider ou non de modifier la répartition du temps qu'ils consacrent à différents contextes d'activités (famille, travail, loisir, bénévolat). Ensuite, les sujets devaient indiquer quel choix ils conseillaient personnellement. Une activation symbolique ou réelle des rapports intersexes était effectuée. Les résultats montrent : 1) que les conseils en faveur de l'individualisme " relationnel » ou " de marché » sont présents chez les sujets des deux sexes ; 2) que l'activation des rapports intersexes modifie davantage les conseils des femmes que ceux des hommes, cette activation s'associant à des conseils plus fréquents, en faveur de la famille et du travail chez les femmes, en faveur des loisirs chez les hommes.
\end{abstract}

(C) 2006 Société française de psychologie. Publié par Elsevier SAS. Tous droits réservés.

\footnotetext{
${ }^{*}$ Auteur correspondant.

Adresse e-mail : manuel.tostain@unicaen.fr (M. Tostain).
}

1269-1763/\$ - see front matter (C) 2006 Société française de psychologie. Publié par Elsevier SAS. Tous droits réservés. doi:10.1016/j.prps.2006.01.013 


\begin{abstract}
The aim of this paper was to study how the activation of intersexes relations affected the choices of activities linked to "relational" or "market" individualism. Firstly, as in Marro's research (1998), stories were presented in which the protagonists, being female or male, for different reasons (personal, professional, etc.) must decide or not to modify the time allocation that they devote to various activities, such as family, work, leisure, or voluntary help. Next, the subjects had to point out which choice they would personally advise. In addition, an activation of intersexes relations was carried out. The results have shown: 1) that personal advice in favour of "relational" or "market" individualism are found in both sexes ; 2) that the activation of intersexes relations modified more personal advice of female subjects than of male subjects (then more oriented, to family and work for women, to leisure for men).
\end{abstract}

(C) 2006 Société française de psychologie. Publié par Elsevier SAS. Tous droits réservés.

Mots clés : Individualisme " relationnel » et « de marché »; Rôles de sexe ; Comparaisons femmes-hommes ; Activation des rapports intersexes

Keywords: "Relational" or "market" individualism; Sex roles; Men-women comparisons; Activation of intersexes relations

\title{
1. Introduction
}

Les rôles de sexe, c'est-à-dire les comportements attendus et valorisés pour chaque groupe de sexe, alimentent une vision différenciée des deux sexes et contribuent, pour une part importante, au maintien des inégalités entre sexes (Apfelbaum, 1997 ; Deaux et Lafrance, 1998 ; Hurtig et Pichevin, 1997 ; Laufer et al., 2003). L'esprit du temps va pour autant dans le sens d'une évolution des rapports entre sexes qui se manifeste par une dénonciation du caractère socialement arbitraire et inégalitaire des rôles de sexe (Padis et Thery, 2001 ; Vigarello, 1993). Toutefois, bien que la société se veuille plus égalitaire et respectueuse de chaque sexe, les recherches sociologiques montrent qu'il subsiste des écarts importants entre la réalité et ces attentes sociales (Fortino, 2002). Par exemple, dans le travail, persistent des phénomènes de ségrégations verticales (les femmes occupent plus fréquemment des postes au bas de l'échelle sociale et au statut plus précaire) et horizontales (les femmes sont surreprésentées dans le secteur social, tandis que les hommes sont surreprésentés dans le secteur productif Maruani, 2003). À propos de la gestion du temps, qui constitue un élément important de la vie de chacun, les études montrent que les femmes se doivent de consacrer davantage de temps aux tâches domestiques, aux tâches familiales, la quantité de temps personnel, de temps libre étant plus importante pour les hommes (Barrère-Maurisson, 2003). Ces travaux montrent, en particulier, que les femmes doivent davantage que les hommes concilier vie familiale et vie professionnelle, ce qui entraîne des contraintes supplémentaires (Méda, 2002).

Les analyses psychosociales complètent ce tableau. Ainsi, Byron (2005) met en évidence que les femmes avec enfants ont plus de difficultés à gérer les conflits entre famille et travail que les hommes avec enfants (Byron, 2005). D'autre part, les recherches de Eagly (Eagly et Dickman, 1997 ; Eagly et Mladinic, 1994) montrent que les «transgressions » des rôles de sexe ne sont pas envisagées de la même façon selon le sexe des sujets qui les réalisent. Ainsi, pour des métiers de prestiges équivalents, on accepte généralement plus facilement d'un homme, qu'il fasse un métier majoritairement exercé par les femmes, que d'une femme, qu'elle fasse un métier majoritairement exercé par les hommes. Ce type de différence 
manifeste souvent une plus grande latitude accordée aux hommes par rapport aux femmes dans le choix de leurs activités. Dans cette optique, peut-être parce que les femmes sont davantage victimes des inégalités liées aux rôles de sexe, certains travaux soulignent que les femmes ont une vision plus critique et sont plus favorables à la transgression des rôles de sexe que les hommes (Martin et Parker, 1995 ; Tap, 1985).

Par ailleurs, Lorenzi-Cioldi (Clemence et al., 1998 ; Lorenzi-Cioldi, 1988) rappelle que les rapports entre groupes se font toujours dans le cadre d'un univers partagé, d'une grille commune. Dans nos sociétés individualistes, cette grille commune correspond à la valorisation de l'autonomie et de la singularité de chacun. Dans ce cadre, les recherches de LorenziCioldi mettent en évidence que chez les hommes, en tant que groupe dominant, il y a homologie entre la norme sociale et la définition de soi. Ainsi, les hommes se conçoivent comme singuliers, non déterminés par leur appartenance de sexe et puisent leur représentation de soi aussi bien dans les traits qualifiés de « masculins » que dans les traits qualifiés de " féminins », d'où une androgynie psychologique fréquente. En revanche, la situation est plus compliquée pour les femmes, car elles sont confrontées à une tension entre, d'une part, les valeurs dominantes qui prônent la singularité de soi et, d'autre part, une dynamique qui, à l'instar des groupes dominés, est davantage centrée sur une référence communautaire à leur groupe de sexe. Ainsi, les femmes se pensent davantage comme ressemblant aux autres femmes, et leur perception de soi est plus centrée sur les traits qualifiés de « féminins » que celle des hommes n'est centrée sur les traits qualifiés de «masculins ». Pour résumer, on peut dire que les hommes, par rapport aux femmes, peuvent, toutes choses égales par ailleurs, plus facilement adopter des comportements ne se référant pas à leur groupe de sexe, ont plus de liberté dans le choix de leurs activités et bénéficient de plus de temps libre, de temps personnel (de Singly, 1993).

Ces recherches, qui s'inscrivent dans le cadre d'une analyse en termes de rapports de domination (Bourdieu, 1998), ne doivent pas cependant conduire à une lecture uniformisante des valeurs et des choix des individus. D'abord, la culture et les dynamiques d'un groupe dominé ne sont pas totalement réductibles à ces rapports de domination. De plus, à l'intérieur de chaque groupe, les attitudes et les comportements peuvent varier de façon importante d'un individu à l'autre. Dans ce cadre, signalons que différents enjeux peuvent coexister. Par exemple, une femme peut investir la famille, non pas uniquement en raison d'une contrainte liée à son identité de femme et de mère, mais aussi car elle considère que la relation à ses enfants renvoie à une envie personnelle forte ${ }^{1}$. Ensuite, il faut se rappeler, que dans le champ social, coexistent des valeurs potentiellement contradictoires qui traversent les deux groupes de sexe. Dans nos sociétés à l'idéologie individualiste, où chacun est censé construire sa vie par le biais de choix dictés par des motivations personnelles, de Singly (2000, 2001) rappelle que les individus des deux sexes sont confrontés à deux types d'individualismes. D'une part, un individualisme que cet auteur appelle de marché, centré sur l'identité statutaire, et qui met en avant des valeurs telles que le pouvoir, l'esprit de compétition, et où on valorise la réussite sociale, spécialement professionnelle. Mais en même temps, il existe un individualisme que de Singly qualifie « de relationnel », centré sur l'intimité avec les autres : réussir sa vie dans ce cadre, c'est avoir une vie affective importante, des liens

\footnotetext{
${ }^{1}$ Bien entendu, il s'agit ici de prendre de nombreuses précautions pour ne pas tomber dans ce que Grignon et Passeron (1989) appellent la logique misérabiliste, qui consiste à magnifier les choix des sujets en position de domination ou qui consiste à inverser les rapports de domination dans l'imaginaire.
} 
forts avec ses enfants ou ses amis. Ces deux types d'individualismes, le premier qui est traditionnellement associé et plus accessible aux hommes, le second davantage relié aux femmes, renvoient pour partie, à la distinction classique entre dimensions " instrumentales » et dimensions « expressives » (Parsons et Bales, 1955). Pour de Singly, s'en tenir à l'un ou à l'autre individualisme, donne, pour une personne donnée, une identité tronquée, et jugée comme telle par les autres. Cet auteur rappelle cependant, que si des tensions identitaires existent chez les hommes et les femmes, elles sont plus grandes pour ces dernières. En effet, si on valorise le fait que les hommes développent un individualisme relationnel, les femmes, quand elles s'orientent vers l'individualisme de marché, sont confrontées à des contraintes plus fortes, de nature sociale (par exemple un marché du travail plus inégalitaire pour les femmes) et psychosociale (par exemple les femmes qui réussissent au niveau professionnel peuvent être soumises à des critiques relatives à leur capacité à continuer à jouer leur rôle de mère ou peuvent faire l'objet de questionnements sur leur féminité. Fortino, 2002 ; Lorenzi-Cioldi, 2002 ; de Singly, 2001).

Si le corps social est favorable, à un niveau général, à une plus grande mixité, à un partage des rôles traditionnellement attachés à chaque sexe, on peut se demander si cela reste vrai quand les individus sont confrontés à des situations concrètes où ils doivent faire un choix entre une activité reliée à l'individualisme de marché et une activité reliée à l'individualisme relationnel. Ici, on peut utilement se référer aux travaux de Marro $(1998,1999)$ qui concernent la tolérance à la transgression des rôles de sexe. Dans ses recherches, cet auteur a présenté à des adolescentes et adolescents, des histoires dans lesquelles des protagonistes (femmes ou hommes) étaient confrontés à des alternatives entre des activités qui ressortissaient de différents centres d'intérêts : familiaux (être disponible pour ses enfants) personnels (pratique d'un loisir), professionnels (acceptation d'une promotion) ou collectifs (participation à une activité syndicale). Puis, les sujets devaient indiquer quel choix ils conseilleraient aux différents protagonistes. En se référant aux rôles de sexe traditionnel et aux données statistiques, pour les protagonistes de sexe féminin, les conseils privilégiant les intérêts personnels ou professionnels aux dépens des intérêts familiaux, étaient catégorisés comme une attitude de tolérance à la transgression des rôles de sexe, tandis que pour les protagonistes de sexe masculin, les conseils renvoyant à cette tolérance correspondaient à ceux en faveur d'activités centrées sur les autres (cf. les intérêts familiaux). Selon ce codage, les résultats montrent des attitudes fréquentes de tolérance à la transgression des rôles de sexe. Ainsi, de nombreux sujets conseillent aux protagonistes de sexe féminin le travail quand celui-ci est opposé à la famille et, à l'inverse, pour ce même type d'alternative, conseillent aux protagonistes de sexe masculin la famille. On note cependant que cette tolérance à la transgression des rôles de sexe tend à être un peu plus grande, pour les protagonistes de sexe masculin que pour les protagonistes de sexe féminin, et un peu plus prononcée chez les garçons que chez les filles. En définitive, dans ces situations dilemmes, si on reprend la grille d'analyse de de Singly, l'individualisme relationnel (au travers de conseils en faveur de la famille) semble plus accepté, en tout cas plus recommandé pour les hommes, que la tendance symétrique, l'individualisme de marché (au travers de conseils en faveur du travail) pour les femmes. Un autre résultat important de cette recherche est que les choix conseillés à chaque protagoniste varient en fonction du type d'alternative. Ainsi, par exemple pour les protagonistes de sexe féminin, si on leurs conseille la famille quand celle-ci est opposée au loisir, dans le cas où la famille est opposée à une promotion dans le travail, on leur conseille, ainsi qu'on l'a indiqué, le travail. 


\subsection{Objectifs de l'étude}

L'objectif de la présente étude était de prolonger le travail de Marro, au travers de la grille d'analyse de de Singly, en étudiant l'effet des dynamiques intersexes sur une population de sujets plus âgés. Le travail de Marro (1998) met en évidence la complexité des choix et les tensions identitaires que connaissent les deux sexes mais ne traite pas spécifiquement, du moins au niveau de sa procédure, de ces dynamiques intersexes. On sait que les travaux psychosociaux (Dafflon, 1999 ; Lorenzi-Cioldi et Dafflon, 1999) consacrés aux différences entre sexes mettent en évidence, que les réponses des deux sexes, dans la mesure où elles s'inscrivent dans le cadre de relations intergroupes, peuvent varier de manière significative en fonction des rapports entre sexes introduits dans le dispositif expérimental. Par exemple, LorenziCioldi (1988) montre que lorsqu'on provoque une activation de l'autre groupe de sexe (en le rendant plus visible ou plus présent), les réponses des individus varient en fonction du statut de leur groupe de sexe. Ainsi, il relève que cette activation a pour effet de provoquer des réponses, en termes de rôles de sexe, plus traditionnelles chez les femmes que chez les hommes. Lorenzi-Cioldi (voir aussi Hurtig, 1998) explique cette différence, en considérant que les femmes sont davantage prisonnières de leur identité collective que les hommes. Dans ce cadre, l'activation de l'autre groupe de sexe accentuerait ce phénomène. Les femmes, en tant que groupe dominé, se trouvent questionnées, ce qui provoque un repli sur un registre de réponses plus conformes aux attentes sociales liées à leur sexe d'appartenance. À l'inverse, les hommes, en tant que groupe dominant où la singularité est valorisée, développent dans ce type de situation, des stratégies de présentation de soi ayant pour but de se distinguer de son groupe de sexe (Durand-Delvigne et Duru-Bellat, 1998). Compte tenu de cela, nous avons défini trois conditions expérimentales qui rendent plus ou moins visibles les rapports intersexes. Par rapports intersexes, nous entendons ici les relations entre groupes de sexe qui s'inscrivent dans une dynamique sociale asymétrique entre femmes et hommes.

En ce qui concerne les situations dilemmes proposées, nous avons présenté des activités typiques de l'individualisme de marché (travail) et de l'individualisme relationnel (famille). Nous y avons ajouté deux autres activités qui ne se réduisent pas à l'un ou l'autre individualisme mais auxquelles les sujets peuvent être confrontés dans leur vie quotidienne. Il s'agit de deux activités de temps librement choisis, l'une, le bénévolat, plutôt en rapport avec l'individualisme relationnel car centré sur les autres, l'autre, le loisir, davantage centré sur soi, et pour lequel, statistiquement, les hommes consacrent une part plus importante de leur temps que les femmes (Barrère-Maurisson, 2001). Par ailleurs, nous avons pris comme sujets des étudiants, dans la mesure où il s'agit d'une tranche d'âge où les choix professionnels et familiaux s'inscrivent dans un futur proche.

\section{Méthodologie}

\subsection{Population}

Quatre cent quatre-vingts étudiants en première et deuxième année universitaire (100 étudiantes et 120 étudiants de sciences économiques, 40 étudiantes et 60 étudiants de sciences, 100 étudiantes et 60 étudiants de psychologie), soit 160 femmes et 160 hommes (âge 
moyen $=19,2$ ans ; écart-type $=1,2$ ans ; minimum $=17$ ans, six mois ; maximum $=23$ ans, quatre mois).

\subsection{Matériel}

Cinq histoires correspondant à des dilemmes entre travail, famille, loisir et bénévolat ${ }^{2}$. Chaque histoire comportait deux versions, l'une avec un protagoniste de sexe féminin, l'autre, avec un protagoniste de sexe masculin.

\subsubsection{Histoire 1 (travail note 1 vs famille note 10)}

C'est une dénommée Hélène (ou histoire mise au masculin avec un protagoniste dénommé Alain). Elle est mariée et elle a deux enfants. Elle travaille à $80 \%$ pour se consacrer à ses enfants le mercredi. Durant cette journée, elle fait différentes activités avec eux. Son employeur lui propose une promotion intéressante tant en termes de poste qu'en termes financier. Cette promotion impliquera qu'elle travaille à temps plein. Elle peut la refuser sans conséquences pour son emploi. Elle hésite, car si elle accepte, elle ne pourra plus réserver ses mercredis pour ses enfants.

\subsubsection{Histoire 2 (loisir note 1 vs travail note 10)}

C'est une dénommée Eliane (Hervé). Elle est cadre dans une grande entreprise publique. Elle fait depuis longtemps de l'escalade avec des amis le samedi. Elle apprécie cette activité durant laquelle elle passe de bons moments. Dorénavant, ses amis ne seront disponibles que le mercredi. De ce fait, elle devra se mettre à $80 \%$ pour continuer à les accompagner. Elle hésite car, même si c'est tout à fait possible pour elle de se mettre à temps partiel, ce ne sera pas évident dans son travail, vu son poste de cadre.

\subsubsection{Histoire 3 (travail note 1 vs activité bénévole note 10)}

C'est une dénommée Élisabeth (Pierre) qui travaille dans une entreprise de fabrication de composants électroniques. On lui propose un changement d'affectation pour un poste qui l'intéressait depuis longtemps et qu'elle peut ou non accepter. Elle hésite car elle changera d'horaires et ne pourra plus participer aux activités d'une association de préservation de l'environnement dont elle est l'une des membres fondatrices.

\subsubsection{Histoire 4 (loisir note 1 vs famille note 10)}

C'est une dénommée Pascale (Eric). C'est quelqu'un de sportif et qui aime les activités aquatiques. Par l'association sportive dont elle fait partie, elle a l'opportunité d'aller à Hawaï où elle avait envie de se rendre depuis longtemps. Elle y restera durant les deux mois d'été et elle en profitera principalement pour s'adonner à sa passion, le surf. Elle hésite car elle ne pourra avoir le plaisir de passer ses vacances avec son mari (sa femme) et ses deux enfants qui ne pourront pas faire le voyage avec elle.

\footnotetext{
${ }^{2}$ Pour éviter une tâche trop répétitive pour les sujets, nous n'avons présenté, pour chaque catégorie d'opposition (par exemple entre famille-travail), qu'un seul conflit. On ne prétend donc pas ici rendre compte avec exhaustivité des divers types de conflits auxquels les sujets peuvent être confrontés pour chaque catégorie d'opposition.
} 


\subsubsection{Histoire 5 (activité bénévole note 1 vs famille note 10)}

C'est une dénommée Lucie (Gaspar). Elle est mariée, son mari (sa femme) travaille, et elle a deux enfants qu'elle récupère un jour sur deux à l'école. Elle est par ailleurs membre d'une grande association de défense des consommateurs. On lui propose de faire partie du comité de pilotage et d'organisation de cette association. Cela lui plairait beaucoup. Elle hésite pourtant, car cela impliquera qu'elle soit assez souvent absente le soir et le week-end. Elle ne pourra donc plus s'occuper comme avant de ses enfants, ce qui lui plaît également beaucoup.

\subsection{Procédure}

On introduisait la recherche en indiquant qu'il s'agissait d'une étude sur les choix auxquels on est confronté dans la vie quotidienne. On présentait à chaque sujet les cinq histoires dans une seule version (soit féminine, soit masculine). L'ordre des histoires était aléatoire. À la suite de chaque histoire, les sujets indiquaient quelle solution ils conseillaient à chacun des protagonistes. Pour cela, ils se positionnaient sur une échelle bipolaire comprenant dix échelons. L'extrémité gauche correspondait à un conseil totalement en faveur de la première activité (note 1), l'extrémité droite, à un conseil totalement en faveur de la seconde activité (note 10), le positionnement vers les échelons centraux indiquant un conseil modérément en faveur de la première (note 5) ou de la seconde activité (note 6).

Les sujets étaient répartis aléatoirement selon trois conditions expérimentales. Condition de base 1 « seul »: les sujets, comme dans la recherche de Marro, devaient indiquer leur conseil personnel. Activation des rapports intersexes (conditions 2 et 3 ). Nous nous sommes inspirés de la méthodologie utilisée par Lorenzi-Cioldi (1988) pour provoquer des rapports intersexes. Condition 2. Activation symbolique par évocation préalable de l'autre sexe : avant d'indiquer leur conseil personnel, les sujets devaient imaginer ce qu'une personne de l'autre sexe conseillerait. Condition 3. Activation réelle par coaction préalable des deux sexes : les sujets étaient mis en dyade mixte et devaient donner un conseil commun pour chacune des cinq histoires, puis, individuellement, ils indiquaient leurs conseils personnels.

\subsection{Plan expérimental}

Deux (sexe des protagonistes des histoires : masculin vs féminin) $\times$ trois (condition « seul » vs activation symbolique vs activation réelle) $\times$ deux (sexe des sujets) $=12$ groupes, chaque groupe comprenant 40 sujets (soit 480 sujets).

\section{Hypothèses}

\subsection{Hypothèse 1}

En référence aux travaux de Marro (1998, 1999), les conseils personnels varient selon le type de dilemme. Plus précisément, en fonction des alternatives présentées, ces conseils sont en faveur de l'individualisme de marché (travail) ou de l'individualisme relationnel (famille). 


\subsection{Hypothèse 2}

Compte tenu des évolutions sociales favorables à une plus grande mixité des rôles, on s'attend à ce que l'individualisme relationnel soit présent dans les conseils pour les protagonistes de sexe masculin (première tendance). De même, on s'attend à ce que l'individualisme de marché soit présent dans les conseils pour les protagonistes de sexe féminin (deuxième tendance). Si on se réfère aux travaux de Marro et aux analyses de de Singly, on peut supposer cependant que la première tendance soit plus forte que la deuxième tendance.

\subsection{Hypothèse 3}

En référence aux analyses de Hurtig (1998) et de Lorenzi-Cioldi (1988) l'activation symbolique ou réelle de l'autre sexe a pour conséquence d'orienter davantage les femmes que les hommes vers des réponses traditionnelles en termes de rôles de sexe.

\section{Résultats}

Comme dans l'étude de Marro (1998), la distribution des scores sur les échelles est de nature bimodale (se concentrant vers chaque extrémité). Cela correspond à un fonctionnement dichotomique des sujets qui, généralement, choisissent de manière assez tranchée une des deux propositions de l'alternative (notes situées vers les extrêmes). Nous n'avons donc pas utilisé l'analyse de la variance, et avons procédé, à la place, à des calculs de $\chi 2^{3}$. Nous avons également calculé des coefficients d'associations ( $V$ de Cramer) pour estimer la force des liens entre variables.

\subsection{Condition de base 1 «seul»}

Préalable : si on se réfère au nombre de conseils donnés en faveur de telle ou telle activité dans la situation de base, on constate, tout sexe des personnes interrogées et tout sexe des protagonistes confondus, que les conseils les plus fréquents se dirigent vers la famille : sur les trois histoires qui opposent la famille à une autre activité, 62,7 \% des conseils sont en faveur de la famille (301/480 conseils). Le loisir, qui était évoqué dans deux histoires, arrive en second avec 53,35\% des conseils (170/320 conseils), puis le travail $(220 / 480=45,8 \%$ ), et enfin le bénévolat $(109 / 320=34 \%$ ) (septième colonne du Tableau 1). Par ailleurs, indépendamment du sexe des personnes interrogées, si on prend en compte le sexe des protagonistes (troisième et sixième colonne Tableau 1), on constate qu'il y a plus de sujets qui conseillent le travail aux protagonistes de sexe féminin (66\% des sujets) qu'aux protagonistes de sexe masculin (46\% des sujets) quand celui-ci est opposé au loisir (cf. histoire 2$)^{4}$. Une différence

\footnotetext{
${ }^{3}$ Nous avons comparé (par des $\chi 2$ ) les résultats en fonction de la filière des étudiants, partant de l'idée que les étudiants, notamment en économie, seraient peut-être plus centrés sur des choix favorisant la réussite professionnelle, tandis que les étudiants en psychologie seraient peut-être plus sensibles aux choix qui mettent en avant des dimensions plus affectives (familiales par exemple). Nous ne tiendrons pas compte de ce critère car nous n'avons pas constaté de différences selon le type d'études suivis par les étudiants.

${ }^{4}$ Histoire $2 \chi^{2}(1)=6,50 ; p<0,01 . V$ de Cramer $=0,20$.
} 
Tableau 1

Répartition des conseils pour chaque alternative en fonction du sexe des protagonistes et du sexe des sujets en condition $1 \ll$ seul» (en pourcentages)

\begin{tabular}{|c|c|c|c|c|c|c|c|c|}
\hline & & \multicolumn{3}{|c|}{ Protagonistes de sexe féminin } & \multicolumn{3}{|c|}{ Protagonistes de sexe masculin } & \multirow{3}{*}{$\begin{array}{l}\text { Prota- } \\
\text { gonistes } \\
\text { féminins } \\
+ \text { masculins } \\
\text { Total } \\
\text { général } \\
\text { Colonne } 7 \\
(\%)\end{array}$} \\
\hline & & Femmes $^{\mathrm{a}}$ & Hommes $^{\mathrm{a}}$ & Total & Femmes & Hommes & Total & \\
\hline & & $\begin{array}{l}\text { Colonne } 1 \\
(\%)\end{array}$ & $\begin{array}{l}\text { Colonne } 2 \\
(\%)\end{array}$ & $\begin{array}{l}\text { Colonne } 3 \\
(\%)\end{array}$ & $\begin{array}{l}\text { Colonne } 4 \\
(\%)\end{array}$ & $\begin{array}{l}\text { Colonne } 5 \\
(\%)\end{array}$ & $\begin{array}{l}\text { Colonne } 6 \\
(\%)\end{array}$ & \\
\hline \multirow[t]{4}{*}{ Histoire 1} & Famille & $82,5 \%{ }^{b}$ & 50 & 66,25 & 90 & 77,5 & 83,75 & 75 \\
\hline & & $(n=33)$ & $(n=20)$ & $(n=53)$ & $(n=36)$ & $(n=31)$ & $(n=67)$ & $(n=120)$ \\
\hline & Travail & 17,5 & 50 & 33,75 & 10 & 22,5 & 16,25 & 25 \\
\hline & & $(n=7)$ & $(n=20)$ & $(n=27)$ & $(n=4)$ & $(n=9)$ & $(n=13)$ & $(n=40)$ \\
\hline \multirow[t]{2}{*}{ Histoire 2} & Loisir & 25 & 42,5 & 33,75 & 70 & 37,5 & 53,75 & 43,75 \\
\hline & Travail & 75 & 57,7 & 66,25 & 30 & 62,5 & 46,25 & 56,25 \\
\hline \multirow[t]{2}{*}{ Histoire 3} & Bénévolat & 45 & 30 & 37,5 & 70 & 40 & 55 & 46,25 \\
\hline & Travail & 55 & 70 & 62,5 & 30 & 60 & 45 & 53,75 \\
\hline \multirow[t]{2}{*}{ Histoire 4} & Loisir & 90 & 62,5 & 76,25 & 65 & 42,5 & 53,75 & 65 \\
\hline & Famille & 10 & 37,5 & 23,75 & 35 & 57,5 & 46,25 & 35 \\
\hline \multirow[t]{2}{*}{ Histoire 5} & Bénévolat & 22,5 & 27,5 & 25 & 12,5 & 25 & 18,75 & 21,88 \\
\hline & Famille & 77,5 & 72,5 & 75 & 87,7 & 75 & 81,25 & 78,12 \\
\hline
\end{tabular}

a 40 sujets de chaque sexe par histoire.

${ }^{\mathrm{b}}$ pour chaque histoire, pourcentage en colonne.

qui va dans le même sens se dégage pour l'histoire 3 où le travail était opposé au bénévolat (pourcentage de sujets en faveur du travail : 1) pour les protagonistes féminins : $63 \%$; pour les protagonistes masculins : $45 \%)^{5}$. Il y a également plus de sujets en faveur du loisir pour les protagonistes féminins ( $76 \%$ des sujets) que pour les protagonistes masculins (54\% des sujets) quand le loisir est opposé à la famille (cf. histoire 4$)^{6}$. Le coefficient d'association montre que ce lien entre sexe des protagonistes et type de choix est d'ailleurs très fort pour cette histoire $(V=0,91)$. À l'inverse, il y a davantage de sujets qui conseillent la famille aux protagonistes de sexe masculin (84\% des sujets) qu'aux protagonistes de sexe féminin (66\% des sujets) quand celle-ci est opposée au travail (cf. histoire 1) ${ }^{7}$. En revanche, pour 1'histoire 5 , qui opposait famille et bénévolat, il n'y a pas de différences entre les protagonistes de sexes féminin et masculin ( $\chi^{2}$ non significatif). Autrement dit, on met plus l'accent, sur le travail et le loisir pour les protagonistes de sexe féminin, sur la famille pour les protagonistes de sexe masculin.

\subsubsection{Protagonistes de sexe féminin}

Concernant les histoires impliquant des protagonistes de sexe féminin, si on ne tient pas compte pour l'instant du sexe des sujets interrogés, les calculs des $\chi^{2}$ montrent que les conseils majoritaires pour telle ou telle activité varient selon les termes des alternatives et qu'il n'y a pas de hiérarchie stable (troisième colonne Tableau 1). Ainsi, si la famille est davantage conseillée quand elle est opposée au bénévolat (cf. histoire 5 où $75 \%$ des sujets

\footnotetext{
${ }^{5}$ Histoire $3 \chi^{2}(1)=4,92 ; p<0,02 . V$ de Cramer $=0,17$.

${ }^{6}$ Histoire $4 \chi^{2}(1)=134,94 ; p<0,0001 . V$ de Cramer $=0,91$.

${ }^{7}$ Histoire $1 \chi^{2}(1)=6,53 ; p<0,01 . V$ de Cramer $=0,20$.
} 
choisissent la famille) $)^{8}$ ou au travail (cf. histoire 1 où $66 \%$ des sujets choisissent la famille) ${ }^{9}$, le travail est davantage choisi que le bénévolat (cf. histoire 3 où $62 \%$ des sujets choisissent le travail $)^{10}$ et que le loisir (cf. histoire 2 où $62 \%$ des sujets choisissent le travail) ${ }^{11}$. En revanche, on note que le loisir est pourtant bien davantage conseillé que la famille à l'histoire 4 (respectivement 76 et $24 \%)^{12}$. Si on tient compte du sexe des sujets (première et deuxième colonne Tableau 1), on remarque que majoritairement, à l'histoire 1, les femmes privilégient la famille ( $83 \%$ des sujets) au travail (17\% des sujets), ce qui n'est pas le cas des hommes qui sont aussi nombreux à conseiller l'une (50\% des sujets) et l'autre (50\% des sujets $)^{13}$. Il y a également davantage de femmes que d'hommes qui conseillent le loisir quand le choix opposé est la famille (cf. histoire 4. choix en faveur du loisir. Femmes : $90 \%$; hommes : $62 \%)^{14}$. En revanche, pour les autres alternatives (cf. histoires 2, 3 et 5), il n'y a pas de variation en fonction du sexe des sujets ( $\chi 2$ non significatifs). En résumé, les sujets privilégient le travail uniquement quand l'autre terme de l'alternative n'est pas la famille, la famille et les loisirs étant conseillés plus fréquemment par les femmes que par les hommes.

\subsubsection{Protagonistes de sexe masculin}

On constate que les deux histoires où se dégagent des majorités (qui sont d'ailleurs très fortes), correspondent à un choix en faveur de la famille (sixième colonne Tableau 1). Il s'agit de l'histoire 1 ( $84 \%$ des sujets pour la famille $)^{15}$, et de l'histoire 5 ( $81 \%$ des sujets pour la famille) ${ }^{16}$. En revanche, pour les trois autres histoires proposées, il n'y a pas de conseils majoritaires qui se dégagent. L'analyse selon le sexe des sujets met en évidence que l'absence de conseils majoritaires pour les histoires 2, 3 et 4 masque en fait des écarts entre les conseils majoritaires des femmes et les conseils majoritaires des hommes qui, pour ces trois histoires, vont dans des directions opposées. Le détail des résultats (quatrième et cinquième colonne Tableau 1) montre, à l'histoire 2, que la majorité des femmes (70\%) conseillent le loisir, la majorité des hommes (62\%) le travail ${ }^{17}$. À l'histoire 4 , le loisir est plus souvent conseillé par les femmes (65\% des sujets), la famille plus souvent par les hommes $(58 \% \text { des sujets })^{18}$. Pour l'histoire 3 enfin, les femmes sont plus nombreuses à conseiller le bénévolat (70\% des sujets), une majorité des hommes conseillant, au contraire, le travail $(60 \% \text { des sujets })^{19}$. En résumé, pour les protagonistes de sexe masculin, on insiste plus fréquemment sur la famille, ce conseil recueillant les deux majorités les plus fortes (cf. histoires 1 et 5) et, d'autre part, les femmes mettent davantage en avant des activités qui résultent d'un réel libre choix (bénévolat, loisir) que les hommes (qui recommandent plus souvent le travail et la famille).

\footnotetext{
${ }^{8}$ Histoire $5 \chi 2(1)=19,01 ; p<0,0001$.

${ }^{9}$ Histoire $1 \chi 2(1)=7,81 ; p<0,005$.

${ }^{10}$ Histoire $3 \chi^{2}(1)=4,51 ; p<0,03$.

${ }^{11}$ Histoire $2 \chi^{2}(1)=7,81 ; p<0,005$.

${ }_{12}$ Histoire $4 \chi^{2}(1)=21,01 ; p<0,0001$.

${ }^{13}$ Histoire $1 \chi^{2}(1)=9,44 ; p<0,002 . V$ de Cramer $=0,49$.

${ }^{14}$ Histoire $4 \chi 2(1)=8,35 ; p<0,003$. $V$ de Cramer $=0,45$.

${ }^{15}$ Histoire $1 \chi^{2}(1)=35,11 ; p<0,0001$.

${ }^{16}$ Histoire $5 \chi^{2}(1)=30,01 ; p<0,0001$.

${ }^{17}$ Histoire $2 \chi^{2}(1)=8,49 ; p<0,003 . V$ de Cramer $=0,46$.

${ }^{18}$ Histoire $4 \chi^{2}(1)=4,073 ; p<0,043$. $V$ de Cramer $=0,31$.

${ }^{19}$ Histoire $3 \chi 2(1)=7,23 ; p<0,007$. $V$ de Cramer $=0,42$.
} 
Tableau 2

Répartition des conseils chez les femmes, pour chaque alternative, en fonction du sexe des protagonistes et de la condition expérimentale (en pourcentages)

\begin{tabular}{|c|c|c|c|c|c|c|c|}
\hline & & \multicolumn{3}{|c|}{ Protagonistes de sexe féminin } & \multicolumn{3}{|c|}{ Protagonistes de sexe masculin } \\
\hline & & Condition 1 & $\begin{array}{l}\text { Condition } 2 \\
(\%)\end{array}$ & $\begin{array}{l}\text { Condition } 3 \\
(\%)\end{array}$ & $\begin{array}{l}\text { Condition } 1 \\
(\%)\end{array}$ & $\begin{array}{l}\text { Condition } 2 \\
(\%)\end{array}$ & $\begin{array}{l}\text { Condition } 3 \\
(\%)\end{array}$ \\
\hline & & $\begin{array}{l}\text { Colonne } 1 \\
(\%)\end{array}$ & $\begin{array}{l}\text { Colonne } 2 \\
(\%)\end{array}$ & $\begin{array}{l}\text { Colonne } 3 \\
(\%)\end{array}$ & $\begin{array}{l}\text { Colonne } 4 \\
(\%)\end{array}$ & $\begin{array}{l}\text { Colonne } 5 \\
(\%)\end{array}$ & $\begin{array}{l}\text { Colonne } 6 \\
(\%)\end{array}$ \\
\hline \multirow[t]{2}{*}{ Histoire $1^{\mathrm{a}}$} & Famille & 82,5 & 70 & 52,5 & 90 & 72,5 & 47,5 \\
\hline & Travail & 17,5 & 30 & 47,5 & 10 & 27,5 & 52,5 \\
\hline \multirow[t]{2}{*}{ Histoire 2} & Loisir & 25 & 27,5 & 30 & 70 & 42,5 & 35 \\
\hline & Travail & 75 & 72,5 & 70 & 30 & 57,5 & 65 \\
\hline \multirow[t]{2}{*}{ Histoire 3} & Bénévolat & 45 & 35 & 17,5 & 70 & 27,5 & 25 \\
\hline & Travail & 35 & 65 & 82,5 & 30 & 72,5 & 75 \\
\hline \multirow[t]{2}{*}{ Histoire 4} & Loisir & 90 & 62,5 & 35 & 65 & 40 & 20 \\
\hline & Famille & 10 & 37,5 & 65 & 35 & 60 & 80 \\
\hline \multirow[t]{2}{*}{ Histoire 5} & Bénévolat & 22,5 & 20 & 15 & 12,5 & 10 & 17,5 \\
\hline & Famille & 77,5 & 80 & 85 & 87,5 & 90 & 82,5 \\
\hline
\end{tabular}

a 40 sujets par condition expérimentale, pour chaque histoire.

\subsection{Influence de l'activation des rapports intersexes sur les réponses des femmes et des hommes}

\subsubsection{Les femmes}

On remarque une modification des réponses selon les conditions expérimentales. Pour ce qui est des protagonistes de sexe féminin, on constate que plus les rapports intersexes sont activés dans le dispositif expérimental (passage de la condition 1 《seul », à la condition 2 activation symbolique de l'autre sexe, à la condition 3 activation réelle de l'autre sexe), et plus les conseils des femmes s'orientent vers le travail quand celui-ci est à la base (en condition 1 « seul ») opposé à la famille ou au bénévolat (colonne 1 à 3 Tableau 2). Il s'agit respectivement des histoires 1 (où le pourcentage de sujets en faveur du travail passe de 35 à $83 \%)^{20}$ et 3 (dans ce cas, ce pourcentage passe de 18 à $\left.48 \%\right)^{21}$. On observe égale-

Tableau 3

Répartition des conseils chez les hommes, pour chaque alternative, en fonction du sexe des protagonistes et de la condition expérimentale (en pourcentages)

\begin{tabular}{|c|c|c|c|c|c|c|c|}
\hline & & \multicolumn{3}{|c|}{ Protagonistes de sexe féminin } & \multicolumn{3}{|c|}{ Protagonistes de sexe masculin } \\
\hline & & Condition & Condition & Condition & Condition & Condition & Condition 3 \\
\hline & & 1 & 2 & 3 & 1 & 2 & \\
\hline & & $\begin{array}{l}\text { Colonne } 1 \\
(\%)\end{array}$ & $\begin{array}{l}\text { Colonne } 2 \\
(\%)\end{array}$ & $\begin{array}{l}\text { Colonne } 3 \\
(\%)\end{array}$ & $\begin{array}{l}\text { Colonne } 4 \\
(\%)\end{array}$ & $\begin{array}{l}\text { Colonne } 5 \\
(\%)\end{array}$ & $\begin{array}{l}\text { Colonne } 6 \\
(\%)\end{array}$ \\
\hline \multirow[t]{2}{*}{ Histoire $1^{\mathrm{a}}$} & Famille & 50 & 60 & 52,5 & 77,5 & 60 & 65 \\
\hline & Travail & 50 & 40 & 47,5 & 22,5 & 40 & 35 \\
\hline \multirow[t]{2}{*}{ Histoire 2} & Loisir & 42,5 & 40 & 35 & 37,5 & 52,5 & 72,5 \\
\hline & Travail & 57,5 & 60 & 65 & 62,5 & 47,5 & 27,5 \\
\hline \multirow[t]{2}{*}{ Histoire 3} & Bénévolat & 30 & 15 & 22,5 & 40 & 32,5 & 35 \\
\hline & Travail & 70 & 85 & 77,5 & 60 & 67,5 & 65 \\
\hline \multirow[t]{2}{*}{ Histoire 4} & Loisir & 62,5 & 55 & 42,5 & 42,5 & 60 & 70 \\
\hline & Famille & 37,5 & 45 & 57,5 & 57,5 & 40 & 30 \\
\hline \multirow[t]{2}{*}{ Histoire 5} & Bénévolat & 27,5 & 25 & 22,5 & 25 & 32,5 & 22,5 \\
\hline & Famille & 72,5 & 75 & 77,5 & 75 & 67,5 & 77,5 \\
\hline
\end{tabular}

a 40 sujets par condition expérimentale, pour chaque histoire. 
ment une augmentation significative des conseils en faveur de la famille quand celle-ci est opposée au loisir ${ }^{22}$ (cf. histoire 4 où le pourcentage en faveur de la famille passe de 10 à $65 \%$ ). On note d'ailleurs pour cette histoire, une liaison importante entre le type de choix et l'activation des rapports intersexes. En revanche, pour les histoires 2 et 5, on n'observe pas de changements significatifs. En résumé, l'activation des rapports intersexes a pour conséquence d'orienter davantage de femmes vers des conseils en faveur des activités professionnelles et aussi, vers des conseils en faveur des activités familiales, quand celles-ci sont opposées à un temps personnel, le loisir.

Si on se réfère aux conseils pour les protagonistes de sexe masculin, on constate que l'activation des rapports intersexes se traduit par une augmentation significative des conseils des femmes en faveur du travail (colonne 4 à 6 Tableau 2) : c'est le cas pour les histoires $1^{23}, 2^{24}, 3^{25}$. Dans ces histoires, le pourcentage de sujets en faveur du travail, minoritaire, en condition 1, devient majoritaire en condition 3. L'autre type d'augmentation significative concerne l'histoire 4 où le loisir est de moins en moins souvent conseillé, tandis que la famille devient majoritairement conseillée (le pourcentage de sujets en faveur de la famille passe ainsi de 35 à $80 \%)^{26}$. Quant à l'alternative entre bénévolat et famille (histoire 5), on constate que les conseils évoluent peu et restent majoritairement en faveur de la famille ${ }^{27}$. Autrement dit, l'activation des rapports intersexes a pour conséquence d'orienter plus fréquemment les femmes vers des conseils axés sur le travail et la famille. On retrouve là des tendances assez proches de celles constatées à propos des protagonistes de sexe féminin.

\subsubsection{Les hommes}

On constate que l'activation des rapports intersexes par le dispositif expérimental ne provoque pas de changements particuliers au niveau des conseils des hommes pour les protagonistes de sexe féminin ( $\chi 2$ non significatifs) (colonne 1 à 3 Tableau 3 ). Dans le cas des protagonistes de sexe masculin (colonne 4 à 6 Tableau 3), il y a une variation significative des choix quand on active les rapports intersexes pour les histoires $2^{28}$ et $4^{29}$. Cette variation se manifeste par des conseils plus fréquents en faveur du loisir (qui passent de 38 à $73 \%$ pour l'histoire 2, de 43 à $70 \%$ pour l'histoire 4).

En définitive, si on compare les résultats des femmes et des hommes, on constate que l'activation des rapports intersexes modifie davantage les réponses des femmes que les réponses des hommes. Cette incidence se traduit, pour les femmes, par des conseils plus fréquents en faveur du travail et de la famille. Pour les hommes, cette incidence, qui ne concerne que les protagonistes de sexe masculin, oriente partiellement vers des choix plus hédonistes (cf. loisir).

\footnotetext{
${ }^{20}$ Histoire $1 \chi 2(2)=8,39 ; p<0,01 . V$ de Cramer $=0,26$.

${ }^{21}$ Histoire $3 \chi 2(2)=7,066 ; p<0,02 . V$ de Cramer $=0,24$.

${ }^{22}$ Histoire $4 \chi^{2}(2)=26,05 ; p<0,0001$. $V$ de Cramer $=0,46$

${ }^{23}$ Histoire $1 \chi^{2}(2)=17,37 ; p<0,0002 . V$ de Cramer $=0,38$.

${ }^{24}$ Histoire $2 \chi^{2}(2)=10,87 ; p<0,004 . V$ de Cramer $=0,30$.

${ }^{25}$ Histoire $3 \chi 2(2)=21,19 ; p<0,0001$. $V$ de Cramer $=0,42$.

${ }^{26}$ Histoire $4 \chi^{2}(2)=16,73 ; p<0,0002 . V$ de Cramer $=0,37$.

${ }^{27}$ Histoire $5 \chi^{2}(2)=1,01 ; p<0,60$. ns. $V$ de Cramer $=0,09$.

${ }^{28}$ Histoire $2 \chi^{2}(2)=9,93 ; p<0,007 . V$ de Cramer $=0,28$.

${ }^{29}$ Histoire $4 \chi^{2}(2)=6,34 ; p<0,04$. $V$ de Cramer $=0,22$.
} 


\section{Discussion}

Si on fait une synthèse des différents résultats obtenus dans cette étude, on peut dire d'abord, concernant la situation de base (condition 1 « seul»), que l'individualisme relationnel est un plus présent que l'individualisme de marché, le choix recueillant les conseils les plus nombreux étant la famille. Toutefois, comme dans les recherches de Marro (1998, 1999), et conformément à notre première hypothèse, l'accent mis sur telle ou telle activité dépend des alternatives auxquelles les sujets sont confrontés. Dans ce sens, on constate parfois une non-transitivité des choix : par exemple, pour les protagonistes de sexe féminin, si la famille l'emporte sur le travail (histoire 1) et celui-ci sur les loisirs (histoire 2), les loisirs l'emportent sur la famille (histoire 4). D'autre part, et cela va dans le sens de notre deuxième hypothèse, on constate qu'une majorité de sujets a tendance à valoriser les conseils en rapport avec une évolution des rôles de sexe. Ainsi, pour un certain nombre d'alternatives, des majorités se dégagent, pour les protagonistes de sexe féminin, en faveur du travail et du temps personnel (loisir), pour les protagonistes de sexe masculin, en faveur de la famille. Néanmoins, lorsque les sujets sont confrontés à l'alternative famille contre travail, qui est typique de l'opposition entre individualisme relationnel et de marché, on remarque un phénomène qui comporte une signification différente selon le sexe des sujets. Pour les protagonistes de sexe masculin (et aussi bien de la part des femmes que des hommes), on conseille plus souvent la famille, c'est-à-dire, si on reprend la grille de Marro (1998), un choix plutôt « non conforme » aux rôles de sexe. Pour les protagonistes de sexe féminin, les conseils majoritaires vont également dans le sens de la famille. Dans ce cas néanmoins, cela signifie qu'on conseille plus souvent un choix « conforme » aux rôles de sexe. De plus, ce choix plus fréquent en faveur de la famille pour les protagonistes de sexe féminin, est spécialement dû aux femmes, les hommes se répartissant équitablement entre les deux facettes de l'alternative. Autrement dit, ici, l'accès à l'individualisme relationnel (famille) est plus présent pour les hommes que l'inverse, l'accès à l'individualisme de marché (travail) pour les femmes, ce qui va dans le sens de l'analyse de de Singly (2001).

Par ailleurs, l'activation des rapports intersexes, si elle modifie peu les réponses des hommes interrogés a, en revanche, une incidence importante sur les réponses des femmes. Cette incidence sur les femmes se manifeste par des conseils plus fréquents, non pas forcément pour des choix plus traditionnels comme nous le pensions (cf. hypothèse 3 ), mais pour des orientations plus utiles et ayant une part de contraintes. En l'occurrence, ces choix se font à la fois en faveur de la famille et du travail et ce, tant pour les protagonistes de sexe féminin que masculin. On a l'impression que cette activation des rapports intersexes se traduit, chez les femmes, par des conseils qui dessinent le portrait de femmes et d'hommes actifs qui se doivent de concilier individualisme de marché et individualisme relationnel. En revanche, pour les hommes, cette incidence est plus faible (il ne concerne que les protagonistes de sexe masculin) et va un peu à l'inverse des femmes, puisque l'activation s'associe à des choix plus hédonistes (en faveur des loisirs) et qui présentent la caractéristique d'être peu typés en termes de rôles de sexe. Cette différence entre femmes et hommes en condition activation est délicate à interpréter. À titre d'hypothèse, peut-être que cette activation rend les femmes plus sensibles à leurs désirs d'un partage des rôles au niveau de la famille et du travail entre femmes et hommes. Quant aux hommes, cette activation les met peut-être dans une position qui les incite à recommander des choix qui correspondent à des aspects socialement valorisés, tels que le souci de soi, la singularité, les activités conviviales. Les conseils en 
faveur des loisirs permettent notamment de mettre en avant des goûts personnels que l'on développe dans des relations amicales.

Ces différents résultats mettent en évidence des évolutions en faveur d'une plus grande mixité des rôles de sexe, des individualismes relationnel et de marché. Précisons que ces résultats présentent certaines limites qui doivent nous prémunir de toutes généralisations hâtives. S'agissant d'une première approche des effets des rapports intersexes sur les choix d'activités, nous n'avons envisagé (de manière à ne pas alourdir la passation), pour chaque catégorie d'opposition, qu'un seul type de conflits (par exemple, pour l'opposition travail-famille (cf. histoire 1), il est proposé au protagoniste de travailler davantage et donc de s'occuper moins de sa famille). Or, on peut imaginer d'autres types conflits (pour le travail et la famille, on peut imaginer l'alternative inverse, où la question qui se poserait serait de savoir s'il faut consacrer ou non davantage de temps à sa famille par rapport au travail) ${ }^{30}$. Ensuite, il s'agit de conseils, et il n'est pas sûr, qu'une fois insérés socialement, professionnellement, avec toutes les contraintes et les pesanteurs auxquels ils seront confrontés, nos sujets (ex-étudiant - es) maintiennent de telles attitudes et les traduisent en choix effectifs. Dans ce sens, si les conseils sont assez ouverts, on sait qu'il y a un décalage entre les faits et les idées. Ainsi, si la société, au niveau du discours, valorise la mixité des rôles, les choix occupationnels réels restent assez différents selon les sexes (Barrère-Maurisson, 2003), et généralement ces différences de choix s'inscrivent dans un contexte où la position des femmes est souvent plus défavorable que celle des hommes (Hurtig et Pichevin, 1997). Enfin, il convient de ne pas minimiser ce qui tient, dans ces réponses, à la désirabilité sociale et aux normes d'ouverture et de nondiscrimination entre les sexes (Eagly et Mladinic, 1994). Ces limites posées, ces résultats sont néanmoins plutôt positifs, car on peut penser que ces orientations constituent, pour partie, des guides pour la détermination des choix des individus. Dans une perspective appliquée, il pourrait être intéressant d'envisager d'utiliser ces résultats dans des interventions sur le terrain (école, entreprise) afin de sensibiliser institutions et individus à l'écart qui se manifestent entre l'évolution des aspirations des deux sexes quant aux choix de leurs activités et le maintien du partage traditionnel des occupations entre femmes et hommes. Dans ce sens, cette recherche s'inscrit dans le contexte des revendications pour une plus grande égalité entre femmes et hommes, et on souhaite qu'elle contribue au débat en faveur d'une déspécialisation des rôles de sexe (Méda, 2002).

Pour la suite, il conviendrait de voir dans quelle mesure ces choix sont influencés par le statut familial des répondant (célibataires ou couples avec ou sans enfants) ou leur position dans la hiérarchie sociale (ouvriers, employés, cadres). On pourrait aussi envisager d'étudier les écarts entre les conseils que les individus jugent souhaitables et ceux qu'ils tiennent pour possibles, compte tenu des contraintes auxquelles ils sont confrontés.

\section{Références}

Apfelbaum, E., 1997. La psychologie sociale à l'épreuve des femmes : l'impensé des rapports de domination. Revue Internationale de Psychologie Sociale 10 (2), 153-169.

Barrère-Maurisson, A., 2001. Partage des temps et des tâches dans les ménages. La documentation française, Paris. Barrère-Maurisson, A., 2003. Travail, famille : le nouveau contrat. Gallimard, Paris.

\footnotetext{
${ }^{30}$ De ce fait, les différents types d'opposition n'ont pu être totalement équilibrés, ce qui peut, pour partie, expliquer certaines non-transitivités des choix.
} 
Bourdieu, P., 1998. La domination masculine. Seuil, Paris.

Byron, K., 2005. A meta-analytic review of work-family conflict and its antecedents. Journal of Vocational Behavior 67 (2), 169-193.

Clemence, A., Lorenzi-Cioldi, F., Deschamps, J.C., 1998. Relations de dominations intergroupes. In: Beauvois, J.L., Joule, R.V., Monteil, J.M. (Eds.), Vingt ans de psychologie sociale expérimentale francophone. Presses universitaires de Grenoble, Grenoble, pp. 67-96.

Dafflon, A.C., 1999. Perception d'homogénéité dans les groupes. In: Beauvois, J.L., Dubois, N., Doise, W. (Eds.), La construction sociale de la personne. Presses universitaires de Grenoble. Grenoble, pp. 147-155.

Deaux, K., Lafrance, M., 1998. Gender. In: Fiske, S.T., Gilbert, D.T. (Eds.), The Handbook of social psychology. McGraw-Hill, Boston, pp. 788-827.

Durand-Delvigne, A., Durru-Bellat, M., 1998. Mixité scolaire et construction du genre. In: Mariani, M. (Ed.), Les nouvelles frontières de l'inégalité : hommes et femmes sur le marché du travail. La Découverte, Paris, pp. 83-92.

Eagly, A.H., Mladinic, A., 1994. Are people prejudiced against women? Some answers from research on attitudes, gender stereotypes, and judgments of competence. In: Stroebe, W., Hewstone, M. (Eds.), European Review of Social Psychology, vol. 5. Wiley, London, pp. 1-35.

Eagly, A.H., Dickman, A.B., 1997. The accuracy of gender stereotypes: a dilemma for feminism. Revue Internationale de Psychologie Sociale 10 (2), 11-30.

Fortino, S., 2002. La mixité au travail. La Dispute, Paris.

Grignon, C., Passeron, J.C., 1989. Le savant et le populaire. Misérabilisme et populisme en sociologie et en littérature. Le Seuil, Paris.

Hurtig, M.C., 1998. Catégories de sexe et perception de soi. Connexions 72, 105-119.

Hurtig, M.C., Pichevin, M.F., 1997. Sexe et cognition. In: Leyens, J.P., Beauvois, J.L. (Eds.), L'ère de la cognition. Presses universitaires de Grenoble, Grenoble, pp. 213-224.

Laufer, J., Marry, C., Maruani, M., 2003. Le travail du genre. Les sciences sociales du travail à l'épreuve des différences de sexe. La Découverte, Paris.

Lorenzi-Cioldi, F., 1988. Individus dominants et groupes dominés : images masculines et images féminines. Presses universitaires de Grenoble, Grenoble.

Lorenzi-Cioldi, F., 2002. Les représentations des groupes dominants et dominés : collections et agrégats. Presses universitaires de Grenoble, Grenoble.

Lorenzi-Cioldi, F., Dafflon, A.C., 1999. Rapports entre groupes et identité sociale. In: Beauvois, J.L., Dubois, N., Doise, W. (Eds.), La construction sociale de la personne. Presses universitaires de Grenoble, Grenoble, pp. 131145.

Méda, D., 2002. Le temps des femmes : pour un nouveau partage des rôles. Flammarion, Collection Champs, Paris.

Marro, C., 1998. La tolérance à la transgression des rôles de sexe chez l'adolescent(e). Pratiques Psychologiques 3 , 39-50.

Marro, C., 1999. Différence des sexes et tolérance à la transgression des rôles de sexe. In: Vouillot, F. (Ed.), Filles et garçons à l'école. CNDP, Collection Autrement, Paris, pp. 123-129.

Martin, C.L., Parker, S., 1995. Folk theories about gender and race differences. Personality and Social Psychology Bulletin 21, 45-57.

Maruani, M., 2003. Travail et emploi des femmes. La Découverte, Paris.

Padis, M.O., Thery, I., 2001. L'un et l'autre sexe. Esprit, 273.

Parsons, T., Bales, R.F., 1955. Family, socialization and interaction processes. Free Press, New-York.

Singly de, F., 1993. Les habits neufs de la domination masculine. Esprit 196, 54-64.

Singly, de, F., 2000. Libres ensemble. L'individualisme dans la vie commune. Nathan, Paris.

Singly de, F., 2001. Charges et charmes de la vie privée. In: Laufer, J., Mary, C., Maruani, M. (Eds.), MasculinFéminin. Presses universitaires de France, Paris, pp. 149-167.

Tap, P., 1985. Masculin-féminin chez l'enfant. Privat, Toulouse.

Vigarello, G., 1993. Vers une neutralisation des genres. Esprit 196, 5-8. 